Revista Perspectiva Online: Humanas \& Sociais Aplicadas Julho de 2017, Vol.7, no 19 , p.65-82

ISSN: 2236-8876 (Online)

DOI:10.25242/887671920171173

\title{
DROGADIÇÃO FEMININA NO BRASIL: UMA ANÁLISE EPIDEMIOLÓGICA
}

\author{
Érica Henrique Ribeiro-Andrade ${ }^{1^{*}}$, Mayara Gomes Evangelista ${ }^{2}$, Vitória Santana Chagas ${ }^{2}$, \\ Adilma Márcia Pinto Silva ${ }^{2}$ \& Maria Francisca Teresa Barbosa Barreto ${ }^{2}$
}

\begin{abstract}
RESUMO
RIBEIRO-ANDRADE,E.H.;EVANGELISTA; M.G;CHAGAS,V.S.;SILVA, A.M.P.;BARRETO, M.F.T.B. Drogadição Feminina no Brasil. Perpectivas Online: Humanas \& Sociais Aplicadas, v.7, n.19, p. 65-82, 2017.
\end{abstract}

Esta pesquisa se desenvolveu por uma preocupação com o gênero feminino no que diz respeito à saúde mental e o crescente perigo que o uso indevido de drogas tem representado. O objetivo geral consiste em mapear os dados epidemiológicos sobre a drogadição feminina no Brasil. Contou-se com recursos da pesquisa bibliográfica para apreciar teoricamente os conceitos de Epidemiologia e sobre Drogadição e Gênero Feminino. Realizou-se uma Revisão Integrativa da Literatura na Rede Scielo, desde sua inauguração até o mês de novembro de 2016, utilizando-se a Análise de Conteúdo. Nos resultados identificou-se a existência de bancos de dados que mostraram-se fundamentais para a continuidade da pesquisa, tais como: o banco de dados virtuais do Sistema Único De Saúde, o Levantamento Nacional de Álcool e Drogas (2012), dados do DEPAN (Departamento Penitenciário Nacional, 2014), e o artigo entitulado A saúde de mulheres e o fenômeno das drogas em revistas brasileiras, escrito em 2014 por profissionais de Enfermagem. É inegável o quanto o estudo que ora desenvolveu-se sobre as terminologias e principais conceituações da Epidemiologia, ampliou a possibilidade de novas buscas, partindo de novos termos tanto na literatura impressa como nos sites de artigos indexados.

Palavras-chave: Drogadição Feminina; Epidemiologia; Drogas; Mulher. 


\begin{abstract}
This research is developed by a concern with the feminine gender related to women's mental health and the growing danger that drug misuse has represented. The general objective of this study is to map the epidemiological data about the feminine drug addicition in Brazil. Was used means of bibliographic research to theorize concept o Epidemiology and about Drug addiction and Feminine Gender. Was realized a Integrative literature review on Scielo, since its inauguration until the month of November of 2006, utilizing the criterions of the Analysis of Content (Bardin, 2009). In results, was identificated the existence

fundamentals for continuity of research, like: the bank of virtuals data of the System Unique of Health, the National Survey of Alcohol and Drugs (2012), data of DEPAN (National Penintentiary Department, 2014), and the article Women's health and the phenomenon of drugs in brazilian magazines, written in 2014 for nursing proffesionals. Its undeniable how much the study that was desenvolved about the terminologies and principals concepts of epidemiology, extended the possibility of new searchs, starting of new terms, both in a print literature and on indexed article sites.
\end{abstract} of bank of virtuals data that were shown

KEYWORDS: Feminine drug addiction; Epidemiology; Drugs; Woman.

\footnotetext{
${ }^{1}$ Institutos Superiores de Ensino do CENSA - ISECENSA - Laboratório de Estudos sobre a Drogadição- Rua Salvador Correa, 139, Centro, Campos dos Goytacazes, RJ, CEP: 28035-310, Brasil;

${ }^{2}$ Acadêmicas de Psicologia ISECENSA- Institutos Superiores de Ensino do CENSA - ISECENSA - Rua Salvador Correa, 139, Centro, Campos dos Goytacazes, RJ, CEP: 28035-310, Brasil;

(*)e-mail: ericahribeiro@yahoo.com.br

Data de chegada: 19/04/2017 Aceito para publicação: 09/06/2017
} 


\section{INTRODUÇÃO}

A Psicologia enquanto área do conhecimento tem se debruçado sobre as questões de gênero investigando distinções entre homens e mulheres do que diz respeito a sua subjetividade, sua saúde emocional e os impactos desta para a saúde global dos sujeitos e sua qualidade de vida. A presente pesquisa se desenvolve por uma preocupação com o gênero feminino, no que diz respeito à saúde mental de mulheres e o crescente perigo que o uso indevido de drogas, lícitas ou ilícitas, tem representado para manutenção e controle desta. Entende-se que para que os profissionais de psicologia possam intervir de maneira assertiva diante desta demanda, dados atuais sobre a realidade da drogadição feminina precisam ser clarificados, apresentados. Trata-se da importância que os dados epidemiológicos podem ter como aliados ao mapeamento e intervenção sobre a referida situação.

O objetivo geral desta pesquisa consiste mapear os dados epidemiológicos sobre a drogadição feminina no Brasil. Para tanto, postula-se quatro objetivos específicos, dos quais dois foram alcançados nesta primeira etapa do estudo e são apresentados em seus resultados no presente texto. Foi necessário, neste primeiro momento, levantar teoricamente o conceito de Epidemiologia e circunscrever a questão da drogadição feminina em termos de uma compreensão do que já foi publicado a respeito. $\mathrm{O}$ estudo sobre o Gênero Feminino e sua especificidade, qual seja a Drogadição Feminina, deve ser vinculado a um entendimento acerca do que seriam dados epidemiológicos, visualizando uma relação da Epidemiologia com os Estudos sobre a Drogadição Feminina como áreas de apoio recíproco.

\section{METODOLOGIA}

Contou-se com recursos da pesquisa bibliográfica para apreciar teoricamente os conceitos da Epidemiologia. Neste caso o filtros de inclusão foram as publicações dos últimos 5 anos, ainda que de obras publicadas originalmente em outras décadas, contudo, que tivessem sido revisadas e reeditadas neste período.

Quanto à temática Drogadição e Gênero Feminino, utilizou-se a pesquisa bibliográfica para apresentar uma teorização a este respeito, também optando por produções recentes. Todavia, optou-se por um levantamento mais completo do estado da arte, investigando toda a publicação do banco de dados indexado no Scielo, desde sua inauguração até o mês de novembro de 2016. Trata-se desta forma de uma Revisão Integrativa da Literatura. Para tanto, realizou-se o cruzamento dos descritores pertinentes à investigação, quais sejam, Droga, Mulher, Drogadição, Gênero Feminino, Dependência Química, todos em suas derivações singular e plural.

Os dados obtidos na pesquisa sobre Epidemiologia, e também na pesquisa sobre Drogadição Feminina, foram tratados dentro do que diz respeito às duas primeiras etapas da Análise de Conteúdo conforme proposta por Bardin(2009), gerando ainda um banco de dados para futuras análises.

A primeira etapa implica objetivamente em lançar-se ao material bruto coletado, organizando-o para etapas posteriores. O leitor verá que para favorecer esta organização, utilizou-se de recursos didáticos, para apresentação dos dados ao longo deste artigo, como: organogramas, tabelas e gráficos.

Após este exercício, identifica-se os primeiros sentidos do que foi organizado, conferindo os primeiros significados do conteúdo, para somente então, enunciar categorias de informações encontradas nos resultados. É o que apresenta-se no desenvolvimento deste estudo. 


\section{RESULTADOS E DISCUSSÃO}

\section{O Conceito de Epidemiologia}

Segundo Alexandre (2012) o caminho percorrido pela Epidemiologia na história, origina-se da experiência de Hipócrates, com a ideia do cuidado tanto do indivíduo quanto das coletividades na aplicação de medidas de saúde na Grécia antiga.

Para Pereira (2015) a Epidemiologia é o conhecimento abrangente sobre dados que afetam uma coletividade, envolvendo eventos ligados à saúde pública. $\mathrm{O}$ autor destaca que, durante muito tempo a perspectiva ortodoxa deste conceito limitava-se ao estudo de doenças infecciosas, levando a um trabalho quase que maciço da Epidemiologia em ocorrências mais evidentes como o caso dos surtos de doenças infecciosas.

Pode-se dizer que desta forma iniciou se o estudo especifico destes surtos, buscando entender o processo implícito às doenças. Este mesmo autor acredita que este foi o movimento fundamental propulsor de uma mudança de atuação da Epidemiologia, qual seja, partindo da análise de doenças agudas e ampliando para as doenças crônicas, transitando de uma atuação focal em surtos para um acompanhamento permanente.

Isto reforça a descrição de Alexandre (2012) que indica uma maior abrangência da Epidemiologia após a segunda guerra mundial, podendo ser aplicada a doenças não transmissíveis, e não somente a doenças infecciosas como anteriormente.

Esta autora destaca que para a concepção da ideia atual de Epidemiologia, é preciso reconhecer alguns nomes de grande evidência, tais como: Louis René Villermé, médico percursor das análises sobre o processo de adoecimento e seu fator social; William Farr, que estudava, rotulava e mensurava as doenças, tendo como grande interferência os estudos de Villermé; Rudolf Ludwig Karl Virchow, que comprovou que algumas doenças eram agravadas devido aos fatores sociopolíticos; e por último, Louis Pasteur, que ganhou espaço na história da Epidemiologia iniciando estudos sobre bactérias.

Entender o processo percorrido por uma doença, identificar aspectos influentes e intervir, planejar e ajudar em medidas de saúde pública e categorizar doenças, são ações de alcance da Epidemiologia segundo Bellusci (2016). Tais ações coadunam com o que Carvalho e Nascimento et al (2011), entenderam ser o benefício da Epidemiologia à população: um sistema de saúde pública mais adequado por meio de uma poderosa ferramenta de reconhecimento e definição das necessidades emergentes em saúde da coletividade.

Para Maletta (2014) os estudos em Epidemiologia relacionam aspectos, agentes e ambientes que influenciam no desenvolvimento de uma doença em determinada região. Segundo este autor um dos focos da Epidemiologia é o estudo dos fatores que influenciam a forma como uma doença se dá, de acordo com o período e o espaço geográfico, delimitando o público afetado, a quantidade de casos, o tempo, fatores climáticos, idade, sexo, ocupação, entre outros aspectos.

Três são as ações centrais dos estudos epidemiológicos sob o processo de adoecimento em grupos de indivíduos, Rouquayrol (2013), quais sejam: avaliar como determinada doença se propaga, descrever os aspectos marcantes do processo, apresentar propostas para intervenção, vigilância e eliminação de doenças. A autora afirma que a Epidemiologia é orientada pelos acontecimentos em grande escala com as pessoas em sociedade, mas isto não encerra que não se considere o meio individual, ao contrário, aponta a falta de acesso à saúde pelo indivíduo em particular como meio de doenças transmissíveis e não transmissíveis comprometerem sua completude física.

Todavia, é inegável o papel que as relações sociais coexistentes têm no desenvolvimento de doenças ou mesmo no estabelecimento da saúde. Para Rouquayrol (2013), as relações afetam a pessoa, sendo necessário, portanto, que os estudos epidemiológicos considerem a dinâmica do adoecimento e a oscilação da periodicidade de uma doença, em função dos impactos ambientais e populacionais implicados.

Persp. online: hum. \& sociais aplicada., Campos dos Goytacazes, 19 (7), 65-82, 2017

seer.perspectivasonline.com.br 
É possível relacionar este dado, à idéia da doença como sintoma de uma condição social. Nesta ênfase está a base dos estudos da Epidemiologia segundo Carvalho (2016), que acredita que esta área da ciência deve comparar os estados de adoecimento entre diferentes grupos sociais.

Para Bellusci (2016) a Epidemiologia passa constantemente por muitas mudanças, uma vez que a sociedade muda dinamicamente. Dentre estas mudanças registra-se o aumento do acesso à saúde, da expectativa de vida da população, da comunicação global e locomoção facilitada para o mundo inteiro, que consistem em fatores geradores de grandes alterações em nosso meio de vida e em como interagimos com nosso planeta. Bellusci acredita que estes e outros aspectos levam a Epidemiologia a corresponder às demandas e a aprofundar a produção de conhecimento em cada etapa do processo epidemiológico por meio de novas modalidades de estudos e da valorização dos aspectos sociais das pesquisas.

\section{Objetivos da Epidemiologia}

De acordo com Bellusci (2016) os dados epidemiológicos têm o intuito de traçar técnicas para acautelar doenças e servir como roteiro para que os profissionais possam abordar os doentes, baseados em avaliações de frequência, dissipação e outros aspectos da doença. Trata-se, segundo a autora, de estudos que visam a melhoria do bem estar coletivo.

Tornar possível um diagnóstico e definir a amplitude de uma doença, reconhecer prováveis perigos do adoecimento da população e estabelecer possibilidades de intervenção são os objetivos da Epidemiologia para Maletta (2014). A crença é a de que esta ciência pretende apontar agentes, razões e formas de avaliar e pesquisar sobre uma doença, apontando a fase na qual elas se encontram em sua afetação da população, e que aspectos influenciam a doença.

Alexandre (2012) afirma que a investigação epidemiológica é executada, desde que haja anunciação de episódios de doenças ou agravos de distintas naturezas, para que medidas de intervenção possam ser tomadas. Nestes casos, segundo a autora, o objetivo é o de clarificar a origem das infecções, apresentar formas de contágio, apontar indivíduos desprotegidos, atestar diagnósticos, definir particularidades epidemiológicas e principalmente apresentar formas de intervenção e contenção para vedar novos episódios.

Sobre os objetivos da Epidemiologia Rouquayrol (2013) destaca que dentre eles é possível mencionar seu esforço em fornecer uma apresentação do estado atual e periódico de uma doença e descrever a predisposição da mesma avaliando sua dissipação histórica.

Um dos pressupostos gerais da Epidemiologia, segundo Pereira (2015) implica a ideia de que as doenças não surgem aleatoriamente em uma coletividade. Desta forma, fica implícita a noção de que os diferentes agravos se sucedem de diferentes aspectos de influência, e que o reconhecimento destes fatores facilita a intervenção.

\section{Método Científico em Epidemiologia}

Segundo Manso e Alves (2015) quando se trata do estudo epidemiológico é importante destacar parâmetros relevantes no percurso da pesquisa. Esses parâmetros podem incluir uma base estatística, analisando de forma ativa a coletividade. Para os autores, os índices estabelecidos em um estudo epidemiológico são geralmente múltiplos de dez, e normalmente apresentam uma amostra delimitada.

Já para Werneck e Almeida (2016), a validade de uma pesquisa epidemiológica está relacionada a inexistência de falhas na metodologia, e o primor está relacionado a inexistência de outras falhas eventuais, por conseguinte, o pesquisador deve permanecer em estado de alerta a fim de impossibilitar falsas conclusões. Bloch e Coutinho (2016) ao determinar as características do método utilizado em pesquisas epidemiológicas, indicam que a mesma ocorre em função de etapas, a saber : uma teoria inicial que gera uma suposição especulativa, que se transforma em uma suposição praticável, esta será investigada e obterá resultados, esses resultados serão comparados com a teoria inicial.

Persp. online: hum. \& sociais aplicada., Campos dos Goytacazes, 19 (7), 65-82, 2017

seer.perspectivasonline.com.br 
Segundo Maletta (2014) o metodo epidemiológico coletivo se apresenta através de dois momentos. Primeiramente a fase descritiva, na qual se estuda os aspectos de influência por meio de dados quantitativos, para depois haver a uma segunda fase, qual seja, a análise. Já o método clínico, afirma o autor, é especificamente aplicado ao indivíduo doente, com objetivo de intervenção e de explicitar motivos que levaram ao adoecimento.

Rouquayrol (2013) acredita que é graças ao método epidemiológico, que se tem sido possível esclarecer questões sobre o adoecimento, podendo, em alguns casos encontrar explicações para a dinâmica de doenças, antes de origem desconhecida.

\section{$\underline{\text { Tipos de Epidemiologia }}$}

Devido a seu grande crescimento e seus extensivos campos de atuação era esperado que a Epidemiologia se dividisse em várias tipologias. Para Pereira (2015), estas subdivisões expressam diferentes questionamentos do estudo do adoecimento de coletividades e diferentes hipóteses apresentadas.

Entende-se que os vários tipos de nomeações dentro da Epidemiologia enquanto ciência, designam também diferentes métodos de estudos e diferentes objetivos específicos também. Não pretende-se neste artigo apresentar exaustivamente estas tipologias, todavia intenciona-se mencionar alguns tipos de Epidemiologia conhecidos, bem como sua caracterização básica.

Segundo Alexandre (2012) o estudo que verifica como uma doença se dispõe em relação à frequência de uma doença ou agravo em relações a variáveis diversas, como o tempo e o espaço, denominase Epidemiologia Descritiva, tendo como característica a aprimoramento das hipóteses e a descrição das formas de precaução de doenças.

Para Manso e Alves (2015) aspectos como o tempo, espaço e pessoa, tem uma grande influência na relação saúde/doença sendo este o motivo da relevância da Epidemiologia Descritiva. Segundo os autores, este tipo de estudo epidemiológico é bastante recorrente, presente inclusive em leis, como a Lei Orgânica da Saúde, que indica que estudos epidemiológicos precisam ser realizados de acordo com a população, delimitando a coletividade vigente em todo e qualquer aparelho do Sistema Único de Saúde. A referida lei do Conselho Nacional de Saúde ( ${ }^{\circ} 8080$ de 1990) dispõe sobre as condições para promoção, proteção e recuperação da saúde, e trata também da organização e o funcionamento dos serviços correspondentes a estas ações.

Uma importante tipologia nasce, de acordo com Alexandre (2012), por volta dos anos 70, com o intuito de ajudar a atuação da medicina em seu tratamento ao paciente. Surgia então, a Epidemiologia Clínica fundamentando se em processos de reconhecimento das patologias que figuravam nos atendimentos em medicina, contribuindo assim, para o êxito da terapia.

De acordo com Pereira (2015) a Epidemiologia Clínica se baseia no tratamento direto do doente, tornando possível avaliar as circunstâncias as quais levaram o indivíduo a adoecer. Entretanto a atividade clínica, sempre se associou ao trabalho dos profissionais da área de Epidemiologia, sendo esses, inicialmente, também profissionais da medicina.

Este mesmo autor apresenta uma tipologia diversa conhecida como Epidemiologia Social que surgiu de uma rejeição a Epidemiologia Clínica fundamental e valorizava os aspectos sociais em relação à forma de adoecer de determinada coletividade, focando seus estudos em localizações com pouco índice de desenvolvimento. Constituindo-se enquanto uma abordagem interdisciplinar com estudos da Sociologia, a Epidemiologia Social, segundo este autor, ainda é menos acessada do que deveria ser, mas já investiga com seus métodos próprios em diferentes regiões, identificando algumas coletividades mais propensas do que outras para determinadas doenças.

Sustentada, sobretudo, na autenticação dos processos protetores da população, a Epidemiologia Crítica, de acordo com Medeiros et al.(2012), se consolidou como saber científico no século XX, e ficou conhecida como a área do conhecimento que analisa a distribuição e as causas das doenças em indivíduos humanos, contribuindo amplamente para as ações de Saúde Pública e Saúde Coletiva.

Persp. online: hum. \& sociais aplicada., Campos dos Goytacazes, 19 (7), 65-82, 2017

seer.perspectivasonline.com.br 
Breilh (2015), defende a tese de que a Epidemiologia Crítica é a grande evolução que quebra a lógica linear e fragmentada de uma Epidemiologia Clássica onde o individuo fica isolado e cercado por uma série de fatores empíricos e externos. A Epidemiologia Crítica estabelece explicações que obedecem ao modo de definir padrões de Saúde Pública, cruzando a dinâmica da lógica geral da população, maneiras de viver mais ou menos saudáveis desenvolvidas em um determinado espaço e tempo, as práticas de vida distintas dos sujeitos e suas condições bio-psicológicas.

Alexandre (2012) apresenta a descrição da Epidemiologia de Campo, indicando que dentre as modalidades possíveis deste tipo de estudo, o mais utilizado, e exercido com melhor desempenho entre os serviços de saúde é a Investigação de Surto, que seria a utilização de metodologias de análise de questões relacionadas a adoecimentos imprevistos na coletividade. Para a autora, a Investigação de Surtos tem grandes marcos na Saúde Pública atual, como os estudos sobre a AIDS, o H1N1, surtos de cólera, entre outros. Destaca ainda que, a Investigação de Surto necessita de resultados rápidos, devido a afetação da área geográfica em surto, e para tratamento imediato, demandando, portanto, a utilização de métodos simples e eficientes.

O presente trabalho nasceu a partir da crença da possibilidade de haver uma Epidemiologia da Drogadição Feminina, uma vez que acredita-se em vivências bastante distintas da questão da drogadição entre os gêneros. Em apoio a esta hipótese, identifica-se na teoria epidemiológica de Rouquayrol (2013), grande ênfase a importância dos fatores de influência referentes ao gênero do indivíduo exposto ao risco. Para a autora é inegável a distinção fisiológica entre os gêneros, ressaltando as diferenças nas vivencias por cada um dos gêneros no que tange ao adoecer. Assim sendo, a forma como um gênero é visto pela coletividade e como se comporta socialmente, tem grande ascendência sobre as doenças e agravos que o atingem.

\section{Drogadição Feminina}

Segundo estudos do Levantamento Nacional de Álcool e Drogas, publicado no Jornal Folha de São Paulo e divulgado em 2012 pela UNIFESP (Universidade Federal do Estado de São Paulo), num período de seis anos, o índice de mulheres brasileiras que bebem álcool com frequência cresceu 34,5\%, confirmando o alcoolismo feminino como uma questão atual e merecedora da preocupação social.

Dados de pesquisas realizadas pelo DEPAN (Departamento Penitenciário Nacional - INFOPEN Mulheres, 2014), indicam que $68 \%$ das mulheres possuem vínculo penal por conta de envolvimento com o tráfico de drogas, a maioria ocupando uma posição de coadjuvante, ou seja, realizando transporte de droga (avião), e poucas exercendo cargo de gerente do tráfico.

Corroborando a este levantamento, Souza, Oliveira e Nascimento (2014), registram que a realidade da drogadição feminina ainda é pouco discutida na literatura brasileira, mas reconhecem a existência de dados epidemiológicos que apontam o crescimento do número de mulheres presas por conta do narcotráfico e que muitas destas fazem uso de drogas.

A abordagem de Bordin, Figlie e Laranjeira (2004, apud PEREIRA E MARUYAMA, 2014) mostra que a busca para tratamento de dependência feminina se deu a partir da segunda metade do século XX, porém suas demandas não eram atendidas, uma vez que os estudos sobre a drogadição da mulher mostravamse recentes. A dificuldade, segundo os autores, de se efetuar estudos sobre tal tema dá-se, entre outros aspectos, pela recorrência da procura das mulheres a serviços não especializados com queixas vagas sobre seu estado físico, muitas vezes não associando-as ao uso de drogas.

De acordo com Carvalho (2016), o aumento da drogadição feminina se dá por razões como mudanças de valores sociais, tecnológicos, culturais, e maior disponibilidade das substâncias para venda. Além desses fatores, o autor aponta para os problemas familiares, prejuízos nas relações de convivência e afeto, e violência doméstica, como aspectos que também são geradores desse aumento do consumo de drogas por parte das mulheres.

Outros autores também associaram o envolvimento de muitas mulheres com as drogas à falta de estrutura familiar. As grandes tensões vividas pela mulher em função de um histórico familiar de abuso de

Persp. online: hum. \& sociais aplicada., Campos dos Goytacazes, 19 (7), 65-82, 2017

seer.perspectivasonline.com.br 
drogas, as responsabilidades que, de acordo com Blume (1986, apud PEREIRA e MARUYAMA, 2014), são adquiridas a partir do momento em que elas se inserem no mercado de trabalho e os conflitos que essas modificações causam em suas vidas, podem agir como aspectos de risco para o envolvimento destas mulheres com os mais variados tipos de substâncias químicas.

Segundo Souza, Oliveira e Nascimento (2014), o envolvimento de mulheres com homens dependentes com quem mantêm vínculos afetivos e familiares - marido, pai, namorado, irmão - as torna vulneráveis à essa conduta.

Afirmam também que estudos epidemiológicos nacionais apontam para um crescimento de mulheres que consomem tanto substâncias químicas ilícitas quanto lícitas, e que dentre estas últimas existe uma variação significativa que extrapola o uso de tabaco ou álcool. Muitas mulheres são dependentes de remédios antidepressivos, ou fazem uso abusivo de remédios para emagrecer e outras medicações prescritas licitamente.

De acordo com os autores, um levantamento em reportagens de revistas brasileiras apontou que as mulheres, mais que os homens, vem utilizando remédios sem indicação objetiva, para melhorar desconfortos do dia a dia. Registram ainda que muitas utilizam desses medicamentos para manterem-se nos padrões de beleza sociais, e que tais substâncias tendem a ser autoadministradas sem avalições mais criteriosas em função de não serem socialmente considerados como "drogas".

A referida pesquisa, destaca também que muitas mulheres, além do quadro de alcoolismo, apresentam anorexia alcoólica, um transtorno alimentar acoplado à dependência química, que leva a mulher a ingestão de bebidas alcoólicas para minimizar o apetite, tendo como consequência a perda de peso.

Em relação ao sexo feminino, o uso abusivo de drogas traz problemas biológicos mais graves e de agravo mais rápido do que em homens. Em recente pesquisa publicada por Ribeiro-Andrade at al (2016), foi possível encontrar indícios de que de fato a dependência da mulher é diferente da do homem em função, dentre outros aspectos, das diferenças fisiológicas e metabólicas que as tornam mais vulneráveis, não apenas à drogadição em si, mas aos danos causados por esta.

De acordo com Castilho (2013), na prática, os direitos, assim como os papéis sociais entre mulheres e homens são experimentados de maneira diversa, e destaca que para a mulher é muito mais difícil manter laços familiares e sociais mediante ao quadro de Dependência Química.

Nesta mesma direção, Pereira e Maruyama (2014) reconhecem que sobre a mulher ocorre uma maior incidência de situações preconceituosas, e que isto ocorre em decorrência do seu papel social como mãe e dona de casa que deve prezar pelo lar, pelos filhos e marido. Este autores ressaltam que a drogadição feminina, mais contundentemente do que o que ocorre com o gênero masculino, é vista como imprópria e intolerável.

No que diz respeito ao tratatamento para Drogadição Feminina, Bordin, Figlie e Laranjeira, (2004, apud PEREIRA e MARUYAMA, 2014), asseveram que é indispensável pensar que o referido cuidado precisa considerar as particularidades da mulher, sendo adequadamente planejado e dirigido para obter maior efeito terapêutico.

Segundo os autores a abordagem desta problemática deve priorizar muito mais o fato de ser mulher do que ser drogadicta, levando em consideração fatores como: maior demanda de suporte emocional devido ao maior isolamento social e a grande sensibilidade aos efeitos estressantes; complicações médicas; problemas de autoconceito; e questões relacionadas à violência.

$\mathrm{Na}$ tentativa de elucidação deste tema, identificou-se a necessidade de avaliar o estado da arte sobre as características da drogadição feminina, uma vez que tais informações podem facilitar o trabalho de pesquisadores, além de embasar cientificamente instituições especializadas com o tratamento de mulheres drogadictas, e auxiliar a própria mulher na compreensão de sua relação com a droga. Assim, objetivou-se analisar o que vem sendo discutido sobre a drogadição feminina, investigando a base de dados da biblioteca eletrônica Scielo, desde a sua constituição até o inicio de mês de novembro de 2016. Ao realizar o cruzamento dos descritores pertinentes, organizou-se os dados obtidos inicialmente numa perspectiva quantitativa. A partir dos cruzamentos realizados, 555 artigos foram localizados.

Persp. online: hum. \& sociais aplicada., Campos dos Goytacazes, 19 (7), 65-82, 2017

seer.perspectivasonline.com.br 


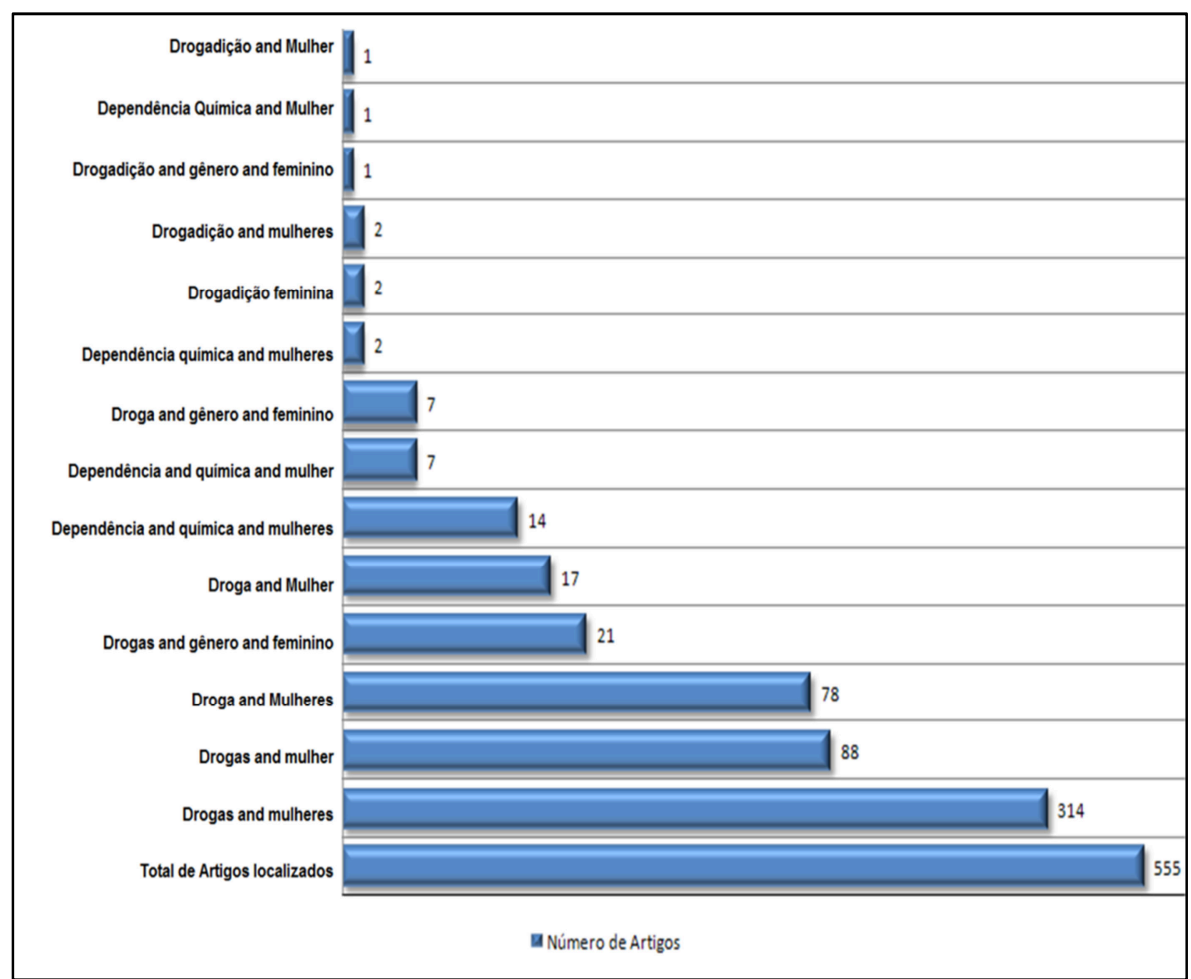

Figura 1: Produção científica sobre Drogadição Feminina na Rede Scielo desde 1999.

Nenhum dos cruzamentos entre os termos envolvendo o descritor "Gênero feminino", gerou algum estudo como resultado, por este motivo não figuram no gráfico acima. Infere-se que tal fato se deva à ainda recente utilização que a academia tem feito do conceito "Gênero feminino".

O mesmo parece ter ocorrido com todos os cruzamentos realizados com o termo "Drogadição" e também o termo "Dependência química", mediante o qual escassos estudos foram identificados.

A maior concentração se deu quando da combinação entre os termos "Drogas/Mulher" com uma variação de ambos os termos no singular e no plural.

Sob os artigos encontrados desenvolveu-se uma subsequente análise qualitativa avaliando-o do ponto de vista dos conteúdos publicados, tendo como referencial metodológico a Análise de Conteúdo de Bardin (2009).

Após excluir os artigos que se repetiam entre os cruzamentos, a avaliação de conteúdo deu-se a partir do critério de real pertinência aos objetivos da pesquisa. Neste caso, manteve-se como resultado somente as publicações que em seu conteúdo tratassem da relação entre a mulher e as drogas, buscando dimensões específicas desta relação.

Muitos artigos que, apesar de terem sido incluídos como resultado dos descritores, não trabalhavam os termos em associação, isto é, alguns mantinham-se como uma pesquisa de gênero sem, no entanto, apresentar a questão da drogadição como um dos seus entornos, e vice versa. Ao término desta primeira analise do conteúdo, restaram 64 artigos ao todo.

Os artigos resultantes desta fase da avaliação constituíram-se em um foco mais específico de leitura, envolvendo desta vez, não apenas os seus títulos e resumos, mas em muitos momentos sua metodologia, suas conclusões e até o artigo por completo. O objetivo desta segunda análise era identificar temáticas recorrentes entre as publicações sobre a drogadição feminina. Isto nos favoreceu ao entendimento de quais os aspectos específicos da referida relação foram privilegiados ao longo do tempo nas publicações identificadas, tendo sido possível nomear nove categorias de conteúdo. 
Dos 64 artigos, vinte e quatro tratavam sobre as Especificidades da Drogadição Feminina, levantando o perfil das usuárias, identificando dados sócio-demográficos, e apontando características da drogadição em questão que eram explicadas em função do gênero.

Também com uma expressão significativa, um grupo de estudos, focou uma dimensão bastante peculiar da experiência feminina, qual seja, a Maternidade. Ora investigando as consequências da drogadição para a puérpera, ora avaliando impactos da drogadição no pré-natal, estes estudos indicaram que o fato de ser mulher e consequentemente responsável fisicamente pela procriação de outros seres, a mulher drogadicta enfrenta desafios bastante diferentes do homem drogadicto que também é pai.

Um grupo menor de artigos, sete ao todo, tinham como um de seus eixos de pesquisa o conceito de Representação Social, e utilizaram-se na maioria das vezes de métodos qualitativos para aferir os significados e a compreensão dos sujeitos de pesquisa acerca da Drogadição Feminina. Cabe ressaltar que entre estes artigos, haviam estudos que interrogavam a mulher sobre questões do universo da adiç̧ão feminina, consistindo em importantes registros sobre a temática à luz do próprio sujeito em questão.

Foi possível registrar a recorrência de estudos que tratavam da dependência química entre as mulheres e diversas Comorbidades. Dentre estes seis estudos, alguns descreviam casos em que a drogadição instalou-se diante de uma doença pré-existente, em outros artigos os casos envolviam novas doenças que foram se instalando em função do quadro de dependência química

Quatro pesquisas tinham como seu objetivo principal estabelecer algum tipo de diferenciação entre a adicção masculina e a feminina, tendo sido nomeados no presente artigo como pesquisas sobre Drogas $e$ Diferenças de Gênero.

De igual forma, quatro outros estudos foram identificados pela sua investigação da relação Drogadição Feminina e o Tráfico. As pesquisas revelam que o envolvimento das mulheres com as drogas tem aberto um novo mercado de trabalho: o tráfico. Todavia os papéis hierárquicos no mundo das drogas obedecem ao que socialmente tem sido inscrito historicamente: a mulher ocupando um papel subalterno.

A questão da sexualidade feminina e sua relação com a Drogadição foi tangenciada por apenas dois estudos, que mencionavam, entre outros aspectos, a prostituição como uma das formas de aquisição da droga quando a dependência da substância já desgastou as soluções financeiras convencionais.

É importante ressaltar que alguns temas identificados nas pesquisas não foram associados em grupos de artigos, pelo menos nesta etapa da análise do material colhido. Isto implica em dizer que pode ocorrer, numa leitura mais pormenorizada destes artigos, estes sejam associados a grupos mencionados anteriormente. Desta forma, criou-se as categorias "Drogas, Mulher e Familia", e "Drogadição Feminina: Crítica aos modelos de tratamento" para identificar temáticas de estudos que embora não tenham se apresentado recorrentemente, implicavam um aspecto muito específico da drogadição feminina. Tão específico, que não foi possível uní-lo a outras categorias.

Os artigos aqui mencionados passarão numa segunda etapa por uma leitura mais atenciosa, identificando outros aspectos dos estudos localizados. Abaixo, segue se uma apresentação da distribuição dos artigos em função das categorias geradas.

Tabela 1: Distribuição dos artigos em função das categorias geradas.

\begin{tabular}{|c|c|}
\hline $\begin{array}{l}\text { CATEGORIAS NOMEADAS NA ANÁLISE DE } \\
\text { CONTEÚDO } \\
\end{array}$ & QUANTIDADE DE ARTIGOS RELACIONADOS \\
\hline $\begin{array}{l}\text { MULHER, DROGA E REPRESENTAÇÃO } \\
\text { SOCIAL.. }\end{array}$ & 07 \\
\hline DROGADIÇÃO E DIFERENÇA DE GÊNERO. & 04 \\
\hline $\begin{array}{c}\text { ESPECIFICIDADES DA DROGADIÇÃO } \\
\text { FEMININA }\end{array}$ & 24 \\
\hline $\begin{array}{l}\text { DROGAS, MULHER E GESTAÇÃO OU } \\
\text { MATERNIDADE }\end{array}$ & $16^{*}$ \\
\hline
\end{tabular}

Persp. online: hum. \& sociais aplicada., Campos dos Goytacazes, 19 (7), 65-82, 2017

seer.perspectivasonline.com.br 


\begin{tabular}{|cc|}
\hline DROGAS, MULHER E SEXUALIDADE & 02 \\
DROGAS, MULHER E FAMÍLIA & 01 \\
MULHER, DROGAS E TRÁFICO & 04 \\
MULHER, DROGADIÇÃO E COMORBIDADES & $06^{*}$ \\
$\begin{array}{c}\text { DROGADIÇÃO FEMININA: CRITICA AOS } \\
\text { MODELOS DE TRATAMENTO }\end{array}$ & 01 \\
\hline
\end{tabular}

(*) Esta categoria possui um artigo em comum com outra categoria

\section{CONCLUSÕES}

O presente levantamento, além de embasar teoricamente a pesquisa que está em desenvolvimento, apontou importantes direções no que diz respeito à busca de dados concretos da Epidemiologia da Drogadição Feminina.

Os artigos restantes dos primeiros filtros aplicados na revisão sistemática consistem em uma primeira fonte de dados sob a qual prosseguir-se-á análise. O objetivo diante destes dados será o de identificar dentre estes artigos selecionados quais deles, de alguma forma, apresentam dados epidemiológicos.

A presente pesquisa deixa como legado a menção que alguns estudos fizeram a bancos de dados virtuais do Sistema Único De Saúde. O Departamento de Informática do Sistema Único de Saúde do Brasil(DATASUS), informa o Portal Saúde do Ministério Brasileiro da Saúde, é um órgão da Secretaria de Gestão Estratégica e Participativa do Ministério da Saúde que foi criado com a finalidade de levantar dados sobre a saúde no Brasil, analisar estes dados, e produzir informações sobre a saúde da população. Entende-se que esta fonte de informação mostrar-se-á como fonte primária de pesquisa nesta próxima etapa.

Alguns estudos mencionados neste artigo citam dados do SIHSUS, Sistema de Informações Hospitalares do SUS. Infere-se que neste banco de dados seja possível identificar informações sobre a recepção de sujeitos drogadictos que procuram os Hospitais de Emergência em decorrência de Episódios de Abstinência, e em função de outras consequências do quadro de dependência química, consistindo também em mais um campo mediante o qual a pesquisa continuará.

Encontrou-se também referências a outras duas possíveis fontes de informação sobre Drogadição, no geral, e sobre Drogadição Feminina de forma mais específica: O SIM (Sistema de Informação Sobre Mortalidade) e o SVE (Sistema de Vigilância Epidemiológica), ambos desenvolvidos pelo Ministério da Saúde.

Um dos artigos analisados, discorre sobre o Levantamento Nacional de Álcool e Drogas, publicado no Jornal Folha de São Paulo e divulgado em 2012 pela UNIFESP (Universidade Federal do Estado de São Paulo), e outro estudo, discorria sobre recente pesquisa realizada pelo do DEPAN (Departamento Penitenciário Nacional) no ano de 2014, por um órgão interno denominado INFOPEN Mulheres. A hipótese alimentada a partir destas duas leituras é que este dois levantamentos mencionados podem apresentar dados pertinentes a presente investigação epidemiológica.

O mesmo ocorreu com o artigo intitulado A saúde de mulheres e o fenômeno das drogas em revistas brasileiras, escrito em 2014 por profissionais de Enfermagem. Estima-se que importantes informações poderão ser encontradas na referida revisão de Márcia Souza, Jean Oliveira e Enilda Nascimento, mediante a qual uma leitura mais detalhada ocorrerá. 
É inegável o quanto a investigação que ora desenvolveu-se sobre as terminologias e principais conceituações da Epidemiologia, ampliou a possibilidade de novas buscas, partindo de novos termos tanto na literatura impressa como nos sites de artigos indexados.

Estes resultados, preliminarmente apresentados permitem ao menos dois enunciados. O primeiro de que o objetivo inicial da pesquisa foi alcançado, qual seja, a conceituação dos eixos temáticos, em virtude do êxito obtido na busca bibliográfica. O segundo enunciado que pode ser elaborado implica o fato de que o próximo estágio da pesquisa mostra-se promissor, uma vez que os Dados Epidemiológicos sobre a Mulher e a Dependência Química começaram a ser vislumbrados.

\section{REFERÊNCIAS}

ALEXANDRE, L. Epidemiologia aplicada nos serviços de saúde. São Paulo. $1^{\mathrm{a}}$ edição. Martinari. 2012.

ALONSO, M.M.C.; MUSAYON, F.Y.O.; DAVID, H.M.S.L. \& GÓMEZ, M.V.M. Consumo de drogas y violencia laboral en mujeres que trabajan, un estudio multicéntrico: México, Perú, Brasil. Rev Latino-am Enfermagem, março-abril; 14(2):155-62,2006. ISSN 0104-1169.

ALVARADO-CHACÓN, R.; SILVA, M.A.I.; GUZMÁN-FACUNDO, F.; SERRANO-CAZORLA, R.I.; \& BENCOMO, A. Significado del consumo de drogas para las adolescentes de la calle, en la ciudad de Valencia, Venezuela. Rev. Latino-Am. Enfermagem, Jun 2011, vol.19, no.spe, p.746-752. ISSN 0104-1169

ANDRADE, A.G.; DUARTE, P.C.A.V.; BARROSO, L.P.; NISHIMURA, R.; ALBERGHINI, D.G.;\& OLIVEIRA, L.G. Use of alcohol and other drugs among Brazilian college students: effects of gender and age. Rev. Bras. Psiquiatr., Oct 2012, vol.34, no.3, p.294-305. ISSN 1516-4446

AQUINO, R.T. R.; VERGUEIRO, C.S.V.; MAGLIARI, M. E.R.; \& FREITAS, T.H. P. Sulfasalazineinduced DRESS syndrome (Drug Rash with Eosinophilia and Systemic Symptoms). São Paulo Med. J., July 2008, vol.126, no.4, p.225-226. ISSN 1516-3180

BARCINSKI, M.. Centralidade de gênero no processo de construção da identidade de mulheres envolvidas na rede do tráfico de drogas. Ciênc. Saúde Coletiva, Dez 2009, vol.14, no.5, p.1843-1853. ISSN 1413-8123

BARCINSKI, M. Protagonismo e vitimização na trajetória de mulheres envolvidas na rede do tráfico de drogas no Rio de Janeiro. Ciênc. Saúde Coletiva, Abr 2009, vol.14, no.2, p.577-586. ISSN 1413-8123

BARDIN, L. Análise de Conteúdo. Lisboa, Portugal; Edições 70, LDA, 2009.

BASSOLS, A.M.S.; BONI, R.; \& PECHANSKY, F. Alcohol, drugs, and risky sexual behavior are related to HIV infection in female adolescents. Rev. Bras.Psiquiatr., Dec 2010, vol.32, no.4, p.361-368. ISSN 15164446

BELLUSCI, S. M. Epidemiologia. São Paulo. Senac. 2016.

BLOCH, K.V.; COUTINHO, E.S.F. Fundamentos da Pesquisa epidemiológica. In: Epidemiologia. 2ed. São Paulo: Editora Atheneu, 2016. p.107-110. Cap.7.

BRASILIANO, S.; HOCHGRAF, P.B. A influência da comorbidade com transtornos alimentares na apresentação de mulheres dependentes de substâncias psicoativas. Rev. Psiquiatr. Clín., 2006, vol.33, no.3, p.134-144. ISSN 0101-6083

Persp. online: hum. \& sociais aplicada., Campos dos Goytacazes, 19 (7), 65-82, 2017

seer.perspectivasonline.com.br 
BREILH, J. Epidemiologia del siglo XXI y ciberespacio:. Repensar la teoría del poder y la determinación de sociais la salud. Rev. bras. epidemiol. , São Paulo, v. 18, n.4, p. 972-982, dezembro de 2015. ISSN1415$790 \mathrm{X}$

CAMPOS, E.A.; REIS, J.G. Representações sobre o uso de álcool por mulheres em tratamento em um centro de referência da cidade de São Paulo - Brasil. Interface (Botucatu), Set 2010, vol.14, no.34, p.539550. ISSN 1414-3283

CARVAlHO, L. F.; DIMENSTEIN, M. O modelo de atenção à saúde e o uso de ansiolíticos entre mulheres..Estud. Psicol. (Natal), Abr 2004, vol.9, no.1, p.121-129. ISSN 1413-294X

CARVALHO, D. (org); NASCIMENTO, E.(org); SILVA, G.; \& GOES, M.A. Vigilância Epidemiológica no Estado de Sergipe - Saberes e tecnologias para implantação de uma política. Livro do Aprendiz 6 / Fundação Estadual de Saúde - Secretaria de Estado da Saúde de Sergipe. - Aracaju: FUNESA, 2011.

CARVALHO, D.M. Epidemiologia- História e Fundamentos. In: Epidemiologia. 2ed. São Paulo: Editora Atheneu, 2016. p.4-11. Cap.1.

CASTILHO, E. Em nome da proteção do cuidado, que formas de sofrimento e exclusão temos produzido? In: CONSELHO FEDERAL DE PSICOLOGIA. Drogas, direitos humanos e laço social. Brasília, 1ed. 160p. Maio, 2013.

CASTILlO, M. M. A.; CAUFIELD, C.; GÓMEZ MEZA, M.V. Consumo de drogas y violencia laboral en mujeres trabajadoras de Monterrey, N. L., México. Rev. Latino-Am. Enfermagem, Dic 2005, vol.13, no.spe2, p.1164-1168. ISSN 0104-1169

CEZARIO, K.; MARIANO, M.R. A percepção de cegos e cegas diante das drogas. Acta Paul. Enferm., 2009, vol.22, no.4, p.404-411. ISSN 0103-2100

CINTRA, A.M.O.; CAIAFFA, W.T.; MINGOTI, S.A. Characteristics of male and female injecting drug users of the AjUDE-Brasil II Project. Cad. Saúde Pública, Apr 2006, vol.22, no.4, p.791-802. ISSN 0102$311 \mathrm{X}$

COSTA, G.M.; SOIBELMAN, M.; ZANCHET, D.L.; COSTA, P. M.; \& SALGADO, C. A.I. Pregnant crack addicts in a psychiatric unit. J. Bras. Psiquiatria, 2012, vol.61, no.1, p.8-12. ISSN 0047-2085

CRUZ, V.D.; OLIVEIRA, M.M.; PINHO, L.B.; COIMBRA, V.C.; CHRISTELLO, K.L.P.; \& OLIVEIRA, J. F. Sociodemographic conditions and patterns of crack use among women. Texto Contexto - Enferm., Dec 2014, vol.23, no.4, p.1068-1076. ISSN 0104-0707

DAVID, H.M. S. L.; CAUFIELD, C. Mudando o foco: um estudo exploratório sobre uso de drogas e violência no trabalho entre mulheres das classes populares da cidade do Rio de Janeiro, Brasil. Rev. LatinoAm. Enfermagem, Dez 2005, vol.13, no.spe2, p.1148-1154. ISSN 0104-1169

DEPARTAMENTO PENINTENCIÁRIO NACIONAL. Levantamento Nacional de informações penitenciárias (INFOPEN - MULHERES). Junho, 2014.

ELBREDER, M. F.; LARANJEIRA, R.; SIQUEIRA, M. M.; \& BARBOSA, D.A. Perfil de mulheres usuárias de álcool em ambulatório especializado em dependência química. J. Bras. Psiquiatr., 2008, vol.57, no.1, p.9-15. ISSN 0047-2085 
FACHINI, A.; FURTADO, E.F.. Diferenças de gênero sobre expectativas do uso de álcool. Rev. Psiquiatr. Clín., 2012, vol.39, no.2, p.68-73. ISSN 0101-6083

FACHINI, A.; FURTADO, E. F. Uso de álcool e expectativas do beber entre universitários: uma análise das diferenças entre os sexos. Psic.: Teor. e Pesq., Dez 2013, vol.29, no.4, p.421-428. ISSN 0102-3772

FERREIRA, V. P.; SILVA, M.A.; NORONHA NETO, C.; FALBO NETO, G. H.; CHAVES, C. V.; \& BELLO, R.P. Prevalência e fatores associados à violência sofrida em mulheres encarceradas por tráfico de drogas no Estado de Pernambuco, Brasil: um estudo transversal. Ciênc. Saúde Coletiva, Jul 2014, vol.19, no.7, p.2255-2264. ISSN 1413-8123

FERTIG, A.; SCHNEIDER, J.F.; OLIVEIRA, G. C.; OLSCHOWSKY, A.; CAMATTA, M.W.; \& PINHO, L. B. Mulheres usuárias de crack: Conhecendo suas histórias de vida. Esc. Anna Nery, Jun 2016, vol.20, no.2, p.310-316. ISSN 1414-8145

FREIRE, K.; PADILHA, P.C.; SAUNDERS, C. Fatores associados ao uso de álcool e cigarro na gestação. Rev. Bras. Ginecol. Obstet., Jul 2009, vol.31, no.7, p.335-341. ISSN 0100-7203

GALERA, S.A.F.; BERNAL ROLDÁN, M.C.; O'BRIEN, B. Women living in a drug (and violence) context: the maternal role. Rev. Latino-Am. Enfermagem, Dec 2005, vol.13, no.spe2, p.1142-1147. ISSN 0104-1169

GIUSTI, J.S; SAÑUDO, A.; SCIVOLETTO, S. Differences in the pattern of drug use between male and female adolescents in treatment.. Rev. Bras. Psiquiatr., June 2002, vol.24, no.2, p.80-82. ISSN 1516-4446

GUIMARÃES, A.B.P.; HOCHGRAF, P.B.; BRASILIANO, S.; \& INGBERMAN, Y. K. Aspectos familiares de meninas adolescentes dependentes de álcool e drogas. Rev. Psiquiatr. Clín., 2009, vol.36, no.2, p.69-74. ISSN 0101-6083

HORTA, R.L.; HORTA, B.L.; PINHEIRO, R.T.; MORALES, B.; \& STREY, M. N.. Tabaco, álcool e outras drogas entre adolescentes em Pelotas, Rio Grande do Sul, Brasil: uma perspectiva de gênero. Cad. Saúde Pública, Abr 2007, vol.23, no.4, p.775-783. ISSN 0102-311X

KASSADA, D. S.; MIASSO, A.I.; WAIDMAN, M.A. P.; \& MARCON, S.S. Prevalence and factors associated with drug use in pregnant women assisted in primary care. Texto Contexto - Enferm.,Sept 2015, vol.24, no.3, p.713-721. ISSN 0104-0707

KASSADA, D.S.; MARCON, S.S.; WAIDMAN, M. A.P. Percepções e práticas de gestantes atendidas na atenção primária frente ao uso de drogas. Esc Anna Nery, Set 2014, vol.18, no.3, p.428-434. ISSN 14148145

LAMOUNIER, J. A; CABRAL, C.M.; OLIVEIRA, B.C.; OLIVEIRA, A. B; OLIVEIRA JR., A. M.; \& SILVA, A. P. A. O uso de medicamentos em puérpuras interfere nas recomendações quanto ao aleitamento materno?. J. Pediatr. (Rio J.), Fev 2002, vol.78, no.1, p.57-61. ISSN 0021-7557

LIMBERGER, J; NASCIMENTO, R. S.; SCHNEIDER, J. A.; \& ANDRETTA, I. Women users of crack: systematic review of Brazilian literature. J. Bras. Psiquiatr., Mar 2016, vol.65, no.1, p.82-88. ISSN 00472085 
LUCCHESE, R.; PARANHOS, D. L.; NETTO, N. S.; VERA, I.; \& SILVA, G.C. Fatores associados ao uso nocivo do tabaco durante a gestação. Acta Paul. Enferm., Jun 2016, vol.29, no.3, p.325-331. ISSN 01032100

MACEDO, F.S.; ROSO, A.; LARA, M.P. Mulheres, saúde e uso de crack: a reprodução do novo racismo na/pela mídia televisiva. Saude Soc., Dez 2015, vol.24, no.4, p.1285-1298. ISSN 0104-1290

MALETTA, C.H. Epidemiologia e Saúde Pública. Belo Horizonte. ND. 2014.Carvalho, H. B. (2010). Epidemiologia. In: Seibel, S. D. Dependência de Drogas. 2 ed. São Paulo: Atheneu.

MANSO, M.E. ALVES, J.C. Manual de Saúde coletiva e Epidemiologia. $1^{\text {a }}$ edição. São Paulo. Martinari. 2015

MARANGONI, S.R.; OLIVEIRA, M. L. F. Fatores desencadeantes do uso de drogas de abuso em mulheres. Texto Contexto - Enferm., Set 2013, vol.22, no.3, p.662-670. ISSN 0104-0707

MEDEIROS, K.T.; MACIEL, S.C.; SOUSA, P. F.; \& VIEIRA, G. L. S. Vivências e Representações sobre o Crack: Um Estudo com Mulheres Usuárias. Psico-USF, Dez 2015, vol.20, no.3, p.517-528. ISSN 1413-8271

MEDEIROS, A. R.P.; LAROCCA, L.M.; CHAVES, M.M.N.; MEIER, M. J.; \& WALL, M.L. A epidemiologia como referencial teórico-metodológico no processo de trabalho do enfermeiro. Rev. esc. enferm. USP, São Paulo, v. 46, n. 6, p. 1519-1523, Dec. 2012 . ISSN 0080-6234.

MELO, M. C.; CORRADI-WEBSTER, C.M. Meanings about mothering by women in treatment for drug use. Estud. psicol. (Campinas), Dec 2016, vol.33, no.4, p.699-709. ISSN 0103-166X

MELO, V. H.; BOTELHO, A.P.M.; MAIA, M.M.M.; CORREA JUNIOR, M. D.; \& PINTO, J.A. Uso de drogas ilícitas por gestantes infectadas pelo HIV. Rev. Bras. Ginecol. Obstet., Dez 2014, vol.36, no.12, p.555-561. ISSN 0100-7203

MENDES, S.U.R.; SILVA, L.F.M.; LOPES, I.F.; MENDES, R. P.R.;\& ROCHA, L.B. M.. Cenário epidemiológico das doenças crônicas não transmissíveis no Tocantins. Anais do $12^{\circ}$.Congresso Brasileiro e Medicina de Família e Comunidade. Pará, 2013.

MESQUITA, M.A. The effects of alcohol in newborns. Einstein (São Paulo), Sept 2010, vol.8, no. 3, p.368375. ISSN 1679-4508

MORAES, C.L.; REICHENHEIM, M.E. Rastreamento de uso de álcool por gestantes de serviços públicos de saúde do Rio de Janeiro. Rev. Saúde Pública, Out 2007, vol.41, no.5, p.695-703. ISSN 00348910

MUSAYÓN, Y.; CAUFIELD, C. Drug consumption and violence in female work Zapallal Lima/Peru. Rev. Latino-Am. Enfermagem, Dec 2005, vol.13, no.spe2, p.1185-1193. ISSN 0104-1169

NÓBREGA, M.P.S.; OLIVEIRA, E.M. Mulheres usuárias de álcool: análise qualitativa. Rev. Saúde Pública, Out 2005, vol.39, no.5, p.816-823. ISSN 0034-8910

NÓBREGA, M. P. S. S.; SIMICH, L.; STRIKE, C.; BRANDS, B.; GIESBRECHT, N.; \& KHENTI, A. Policonsumo simultâneo de drogas entre estudantes de graduação da área de ciências da saúde de uma universidade:implicações de gênero, sociais e legais, Santo André - Brasil. Texto contexto - enferm., 2012, vol.21, no.spe, p.25-33. ISSN 0104-0707 
NUCCI, M.. La píldora anticonceptiva, ¿una droga de "estilo de vida"?: Ensayo sobre el proceso actual de medicalización de la sexualidad. Rev Sex., Salud Soc. (Rio J.), Abr 2012, no.10, p.124-139. ISSN 19846487.

OLIVEIRA, J. F.; PAIVA, M.S.. Vulnerabilidade de mulheres usuárias de drogas ao HIV/AIDS em uma perspectiva de gênero . Esc. Anna Nery, Dez 2007, vol.11, no.4, p.625-631. ISSN 1414-8145

OLIVEIRA, J.F.; MCCALLUM, C. A.; COSTA, H.O.G. Representações sociais de Agentes Comunitários de Saúde acerca do consumo de drogas. Rev. esc. enferm. USP, Set 2010, vol.44, no.3, p.611-618. ISSN 0080-6234

OLIVEIRA, J.F.; NASCIMENTO, E.R.; PAIVA, M.S. Especificidades de usuários(as) de drogas visando uma assistência baseada na heterogeneidade. Esc. Anna Nery, Dez 2007, vol.11, no.4, p.694-698. ISSN $1414-8145$

OLIVEIRA, J.F.; PAIVA, M.S.; VALENTE, C. M.L. The interference of the care context with the visibility of the drug consumption by women. Rev. Latino-Am. Enfermagem, Apr 2007, vol.15, no.2, p.247-252. ISSN 0104-1169

OLIVEIRA, J.F.; PAIVA, M.S.; VALENTE, C. L. M. Representações sociais de profissionais de saúde sobre o consumo de drogas: um olhar numa perspectiva de gênero. Ciênc. saúde coletiva, Jun 2006, vol.11, no.2, p.473-481. ISSN 1413-8123

OLIVEIRA, T.A.; BERSUSA, A.A.S.; SANTOS, T. F.; AQUINO, M.M.A.; \& MARIANI NETO, C. Perinatal Outcomes in Pregnant Women Users of Illegal Drugs. Rev. Bras. Ginecol. Obstet., Apr 2016, vol.38, no.4, p.183-188. ISSN 0100-7203

PEDROSO, R. S.; KESSLER, F.; PECHANSKY, F. Treatment of female and male inpatient crack users: a qualitative study. Trends Psychiatry Psychother., 2013, vol.35, no.1, p.36-45. ISSN 2237-6089

PEREIRA, F.F.B.; MARUYAMA, M. D. G. N. Dependência do álcool em mulheres: aspectos relevantes no tratamento. Uberlândia: FASES, 2014. Monografia.

PEREIRA, M. Epidemiologia Teoria e Prática. Rio de Janeiro. Guanabara Koogan. 2015.

PIMENTEL, G.G.A.; OLIVEIRA, E.R.Netto.; PASTOR, A.P. Significados das práticas corporais no tratamento da dependência química. Interface (Botucatu), Mar 2008, vol.12, no.24, p.61-71. ISSN 14143283

PRADO, M.A.M.; QUEIROZ, I.S. A emergência da politização da intimidade na experiência de mulheres usuárias de drogas. Estud. psicol. (Natal), Ago 2012, vol.17, no.2, p.305-312. ISSN 1413-294X

QUITETE, B.; PAULINO, B.; HAUCK, F.; AGUIAR-NEMER, A.S.; \& SILVA-FONSECA, V.A. Transtorno de estresse pós-traumático e uso de drogas ilícitas em mulheres encarceradas no Rio de Janeiro. Rev. psiquiatr. clín., 2012, vol.39, no.2, p.43-47. ISSN 0101-6083

REIS, L.G.; SILVA, C.J.; TRINDADE, A.; ABRAHÃO, M.; \& SILVA, V.A. Women who smoke and stop during pregnancy: who are they?. Rev. Bras. Saude Mater. Infant., Mar 2008, vol.8, no.2, p.217-221. ISSN 1519-3829 
RIBEIRO, D.V.A.; TURATO, E.R.; AZEVEDO, R.C.S.;.\& CAMPOS, C. J. G. Views on treatment adherence among psychoactive substance-dependent women in the outpatient setting: a qualitative study.Trends Psychiatry Psychother., 2012, vol.34, no.4, p.198-206. ISSN 2237-6089

RIBEIRO-ANDRADE, É.H.; BARRETO, M.F.T.B.; MOTA, M.L., TERRA, M.L.G. Dependência química e gênero: uma leitura da experiência feminina na drogadição. Humanas Sociais \& Aplicadas, [S.1.], v. 6, n. 16, ago. 2016. ISSN 22368876.

ROCHA, P. C.; BRITTO E ALVES, M. T. S.S.; CHAGAS, D. C.; SILVA, A.A.M.; BATISTA, R.F.L.; \& SILVA, R.A. Prevalência e fatores associados ao uso de drogas ilícitas em gestantes da coorte BRISA. Cad. Saúde Pública, 2016, vol.32, no.1. ISSN 0102-311X

ROLDÁN, M.C.B.; GALERA, S.A.F. Perception of the mothering role of women who live in a context of drugs and violence. Rev. Latino-Am. Enfermagem, Dec 2005, vol.13, no.spe2, p.1118-1126. ISSN 01041169

ROUQUAYROL, M.Z.; ALMEIDA FILHO, N. Epidemiologia e saúde. Rio de Janeiro. $7^{\text {a }}$ edição. Medsi. 2013.

SILVA, A.F.; CARBONERA, A.; PRADO, J.A.F.A. A luta de uma mulher contra a dependência química: Um olhar da Gestalt-terapia. Revista IGT na Rede, v. 11, nº 21, 2014. p. 297 - 307.

SILVA, V.N.; D'OLIVEIRA, A.F.; MESQUITA, F. Vulnerabilidade ao HIV entre mulheres usuárias de drogas injetáveis. Rev. Saúde Pública, Dez 2007, vol.41, suppl.2, p.22-30. ISSN 0034-8910

SOUZA, J.G.; LIMA, J.M. B.; SANTOS, R.S. Alcoolismo feminino: subsídios para a prática profissional da enfermagem. Esc. Anna Nery, Dez 2008, vol.12, no.4, p.622-629. ISSN 1414-8145

SOUZA, K.O.J. A pouca visibilidade da mulher brasileira no tráfico de drogas. Psicol. estud., Dez 2009, vol.14, no.4, p.649-657. ISSN 1413-7372

SOUZA, M. R. R.; OLIVEIRA, J. F.; NASCIMENTO, E. R. A saúde de mulheres e o fenômeno das drogas em revistas brasileiras. Texto Contexto Enferm, Florianópolis,v. 23, n.1, p. 92-100, 2014.

SOUZA, M,R,R,; OLIVEIRA, J,F.; NASCIMENTO, E.R.;\& CARVALHO, E.S.S. Droga de corpo!Imagens e representações do corpo feminino em revistas brasileiras. Rev. Gaúcha Enferm., Jun 2013, vol.34, no.2, p.62-69. ISSN 1983-1447

SOUZA, M. R. ROCHA.; OLIVEIRA, J. F.; NASCIMENTO, E.R. Women's health and the phenomenon of drugs in brazilian magazines. Texto contexto - enferm, Mar 2014, vol.23, no.1, p.92-100. ISSN 0104-0707

TRACTENBERG, S.G.;. VIOLA, T.W.;.ROSA, C.S.O.; DONATI, J.M.;.FRANCKE, I. D; PEZZI, J.C.; \& GRASSI-OLIVEIRA, R. Exposição a trauma e transtorno de estresse pós-traumático em usuárias de crack. J. bras. psiquiatr., 2012, vol.61, no.4, p.206-213. ISSN 0047-2085

TRINDADE-SUEDAM, I.K.; KOSTRISCH, L.M.V.; PIMENTA, L.A.F.; NEGRATO, C. A.; FRANZOLIN, S.B.; \& TRINDADE JUNIOR, A.S. Diabetes mellitus and drug abuse during pregnancy and 
the risk for orofacial clefts and related abnormalities. Rev. Latino-Am. Enfermagem, 2016, vol.24. ISSN 0104-1169

VARGAS, D.; SOARES, J.; LEON, E.; PEREIRA, C.F.; \& PONCE, T.D. O primeiro contato com as drogas: análise do prontuário de mulheres atendidas em um serviço especializado. Saúde debate, Set 2015, vol.39, no.106, p.782-791. ISSN 0103-1104

WAGNER, G. A.; STEMPliUK, V. A.; ZILBERMAN, M. L.; BARROSO, L. P.; \& ANDRADE, A. G.Alcohol and drug use among university students: gender differences. Rev. Bras. Psiquiatr., June 2007, vol.29, no.2, p.123-129. ISSN 1516-4446

WERNECK, G.L.; ALMEIDA, L.M. Validade em Estudos Epidemiológicos. In: Epidemiologia. 2ed. São Paulo: Editora Atheneu, 2016. p.199-200. Cap.14.

WHITEHORNE-SMITH, P.; SIMICH, L.; STRIKE, C.; BRANDS, B.; GIESBRECHT, N.; \& KHENTI, A. Gender differences in simultaneous polydrug use among undergraduate students of one university, Kingston - Jamaica. Texto contexto - enferm., 2012, vol.21, no.spe, p.74-78. ISSN 0104-0707

YAMAGUCHI, E. T.; CARDOSO, M.M.S.C.; TORRES, M.L.A.; \& ANDRADE, A.G.D. Drogas de abuso e gravidez. Rev. psiquiatr. clín., 2008, vol.35, suppl.1, p.44-47. ISSN 0101-6083 